Pacific Journal of Mathematic 


\section{KERNEL REPRESENTATIONS OF OPERATORS AND THEIR ADJOINTS}

\section{F. Dennis Sentilles}

If $S$ is a locally compact and Hausdorff space and $A$ is a continuous linear operator from $C_{0}(S)$ into the space $C(T)$ with the supremum norm topology then the Riesz Representation Theorem yields the formula $[A f](x)=\int_{S} f(y) \lambda(x, d y)$, where for each $x \in T \lambda(x, \cdot)$ is a complex-valued regular Borel measure on $S$. More generally a study is made of kernel functions $\lambda$ such that $\int_{S} f(y) \lambda(\cdot, d y) \in C(T)$ for $f$ of compact support on $S$. It is shown that $\lambda(\cdot, E)$ is measurable for each Borel set $E$ and that $\mu(E)=\int_{T} \lambda(x, E) \nu(d x)$ is a regular measure on $S$ yielding the adjoint formula $A^{*} \nu=\mu$. Necessary and sufficient conditions are given on $\lambda$ so that $A^{* *}(C(S)) \subset C(T)$ and that $A^{* *}$ be continuous from $C(S)_{\beta}$ to $C(T)_{\beta}$ when $S$ is paracompact. Furthermore, kernel representations of $\beta$-continuous operators are studied with applications to semi-groups of operators in $C_{0}(S)$ and $C(S)_{\beta}$ when $S$ is locally compact.

We point out that as a consequence of our work the condition (1.7) in the paper by Foguel [7] follows from (1.6) when the space is locally compact and Hausdorff. Further the regularity of the above measure yields the more specific vector-valued measure representation of $A, \mu(E)=\lambda(\cdot, E)$ in the sense of [5, Th. 2, p. 492].

Definition and Notation. If $X$ is a locally compact Hausdorff space we denote by $C(X), C_{0}(X)$ and $C_{c}(X)^{+}$the collection of all bounded continuous complex-valued functions on $X$, those vanishing at infinity, and those nonnegative functions of compact support, respectively. The $\sigma$-algebra of Borel sets is the $\sigma$-algebra generated by the open subsets of $X$. We denote by $M(X)$ the space of bounded regular Borel measures on $X$ with variation norm and by $B(X)$ the space of bounded Borel measurable functions on $X$. Let $M(X)^{+}$denote the nonnegative measures in $M(X)$. We give $B(X), C_{0}(X)$ and $C(X)$ the supremum norm topology and $\|f\|=\sup \{|f(x)|: x \in X\}$.

We wish to consider two further topologies on the space $C(X)$. We denote by $C(X)_{\beta}$ the space $C(X)$ with the locally convex topology defined by the collection of seminorms $P_{\phi}(f)=\|\phi f\|, \phi \in C_{0}(X)$. Buck [1] has shown that $C(X)_{\beta}$ has adjoint or dual space $M(X)$. We denote by $C(X)_{\beta}$, the space $C(X)$ with the locally convex topology whose base of neighborhoods at the origin consists of all convex, balanced, 
absorbent sets $V$ such that for each $r>0$ there is a $\beta$ neighborhood of the origin, $W$, such that $W \cap B_{r} \subset V$ where $B_{r}=\{f \in C(X):\|f\| \leqq r\}$. In a recently submitted paper Dorroh [4] introduces this topology and shows that $C(X)_{\beta^{\prime}}$ has dual $M(X)$ and that $\beta=\beta^{\prime}$ for $X$ a paracompact space. Further results on $C(X)_{\beta}$ and $C(X)_{\beta}$, have been recently obtained by Collins and Dorroh in [2]. A set $H \subset M(X)$ is $\beta$-equicontinuous ( $\sigma^{\prime}$-equicontinuous) if there is a $\beta\left(\beta^{\prime}\right)$ neighborhood of 0 , $W$, such that $\left|\int_{X} f d \mu\right| \leqq 1$ for all $f \in W$ and $\mu \in H$. The $\beta$-equicontinuous sets of $M(X)$ have been characterized by Conway [3] who has shown that $H$ is $\beta$-equicontinuous if and only if $H$ is uniformly bounded and for each $\varepsilon>0$ there is a compact set $K \subset X$ such that the variation of $\mu$ on $X-K$ is less than $\varepsilon$ for all $\mu \in H$. Since $\beta^{\prime}$ is a finer topology than $\beta$ any $\beta$-equicontinuous set is $\beta^{\prime}$-equicontinuous and these are the same when $X$ is paracompact.

Suppose $S$ and $T$ are locally compact and Hausdorff. Let $\Delta$ denote the collection of open sets in $S$ and $\sigma(\Delta)$ the collection of Borel sets. We consider complex-valued functions $\lambda$ defined on $T \times \sigma(\Delta)$ such that $\lambda(x)=\lambda(x, \cdot) \in M(S)$. For brevity we will denote this by $\lambda: T \rightarrow M(S)$. We denote the norm of the measure $\lambda(x)$ by $\|\lambda(x)\|$ and set $\|\lambda\|=$ $\sup \{\|\lambda(x)\|: \mathrm{x} \in T\}$. If $f \in B(S)$ we write $\lambda(f)$ for the function defined by $\lambda(f)(x)=\int_{S} f(y) \lambda(x, d y)$ and $\lambda(\cdot, E)$ is the function whose value at $x$ is $\lambda(x, E)$ for $E \in \sigma(\Delta)$. We let $|\lambda|(x, E)$ be the variation of the measure $\lambda(x, \cdot)$ on the set $E$. We will say that the kernel $\lambda$ satisfies condition $E\left(E^{\prime}\right)$ if $\{\lambda(x): x \in K\}$ is $\beta$-equicontinuous ( $\beta^{\prime}$-continuous) for each compact set $K \subset T$.

Finally we take our topology from [8] and topological vector space terminology from [9]. We make use of the Riesz Representation theorem throughout and in particular its corollary:

$$
|\mu|(U)=\sup \left\{\left|\int f d \mu\right|: f \in C_{c}(S),\|f\| \leqq 1, \operatorname{support}(f) \subset U\right\}
$$

for each open set $U$.

We prove the following theorems.

THEOREM 1. (1) If $\lambda: T \rightarrow M(S)^{+}$and $\lambda(f)$ is lower semi-continuous for each $f \in C_{c}(S)^{+}$then $\lambda(\cdot, E)$ is Borel measurable for each $E \in \sigma(\Delta)$.

(2) If $\lambda: T \rightarrow M(S)$ and $\lambda(f) \in C(T)$ for all $f \in C_{c}(S)$ then $\lambda(\cdot, E)$ and $|\lambda|(\cdot, E)$ are measurable for each $E \in \sigma(\Delta)$.

(3) If $\lambda$ satisfies (1) or (2) and $\|\lambda\|<\infty$ then $\lambda(f) \in B(T)$ for $f \in B(S)$.

THEOREM 2. If $\lambda$ satisfies (3) of Theorem 1 then for each $\nu \in M(T)$ 
the formula $\mu(E)=\int_{T} \lambda(x, E) \nu(d x)$ defines a regular Borel measure on $S$ such that $|\mu|(E) \leqq \int_{T}|\lambda|(x, E)|\nu|(d x)$ and for $f \in B(S)$ we have $\int f d \mu=\int \lambda(f) d \nu$.

THEOREM 3. Suppose $A$ is a continuous linear operator from the space $X$ to the space $Y$ where $X$ denotes $C_{0}(S), C(S)_{\beta}$ or $C(S)_{\beta}$, and $Y$ denotes $C(T), C(T)_{\beta}$ or $C(S)_{\beta^{\prime}}$. Then there is a unique mapping $\lambda: T \rightarrow M(S)$ such that

(1) $A f=\lambda(f)$ for all $f \in X$ and

$$
\|\lambda\|=\sup \{\|A f\|: f \in X,\|f\| \leqq 1\}<\infty \text {. }
$$

(2) The adjoint of $A, A^{*}$, takes $M(T)$ into $M(S)$ and is given by

$$
\left(A^{*} \mu\right)(E)=\int_{T} \lambda(x, E) \mu(d x)
$$

(3) Under the natural imbeddings of $B(S)$ and $B(T)$ into $M(S)$ * and $M(T)^{*}$ respectively we have for $f \in B(S)$

$\lambda(f)=A^{* *} f$ where $A^{* *}$ is the adjoint of $A^{*}$ restricted to $M(T)$ Hence $A^{* *}(B(S)) \subset B(T)$ and $A^{* *}$ defines a continuous extension of $A$ to $B(S)$ into $B(T)$.

TheOREM 4. Let $\lambda: T \rightarrow M(S)$. If $\lambda(f) \in C(T)$ for all $f \in C_{c}(S)$ and $\lambda$ satisfies condition $E^{\prime}$ then $\lambda(f)$ is a continuous function on $T$ for $f \in C(S)$. Conversely, if $S$ is paracompact and $\lambda(f)$ is continuous for $f \in C(S)$ then $\lambda$ satisfies condition $E$.

THEOREM 5. Let $\lambda: T \rightarrow M(S)$ and $A$ the linear operator on $C(S)$ defined by $A f=\lambda(f)$. Then $A$ is a continuous operator from $C(S)_{\beta}$, into $C(T)_{\beta}$ or $C(T)_{\beta}$ if and only if $\|\lambda\|<\infty, \lambda(f) \in C(T)$ for $f \in C_{c}(S)$ and $\lambda$ satisfies condition $E^{\prime}$.

Corollary 1. Let $A: C_{0}(S) \rightarrow Y$ where $Y$ is as in Theorem 3 . Then $A^{* *}$ is a continuous operator from $C(S)_{\beta}$, into $C(T)_{\beta}$, if and only if the kernel $\lambda$ satisfies condition $E^{\prime}$. Moreover $A^{* *}$ is the only extension of $A$ to $C(S)$ given by a kernel and consequently is the only $\beta$ or $\beta^{\prime}$ continuous extension of $A$ to $C(S)$.

Proof of Theorem 1. Let $U$ be an open subset of $S$ and let $\chi$ denote its characteristic function. Since $\lambda(x)$ is regular it follows that $\lambda(x, U)=\sup \left\{\lambda(f)(x): 0 \leqq f \leqq \chi, f \in C_{c}(S)^{+}\right\}$. Since $\lambda(f)$ is lower semicontinuous for each $f \in C_{c}(S)^{+}$, then $\lambda(\cdot, U)$ is lower semi-continuous and hence Borel-measurable. Let $\Sigma$ denote the class of Borel sets $E$ 
for which $\lambda(\cdot, E)$ is measurable. Then $\Sigma$ contains all open sets and is closed under countable unions of mutually disjoint sets $E \in \Sigma$ and, if $A, B \in \Sigma$ and $A \supset B$ then $A-B \in \Sigma$. It now follows from [6, p. 2] that $\Sigma=\sigma(\Delta)$ and (1) is proven.

We now prove (2). If $U$ is an open set then as a consequence of the Riesz Representation Theorem we have

$$
|\lambda|(x, U)=\sup \left\{|\lambda(f)(x)|: f \in C_{c}(S),\|f\|=1 \text { and } \operatorname{support}(f) \subset U\right\}
$$

for each $x \in T$.

This means that $|\lambda|(\cdot, U)$ is lower semi-continuous and as in the proof of (1) that $|\lambda|(\cdot, E)$ is measurable for each Borel set $E$.

We can suppose for the remainder of the proof that $\lambda(x)$ is a real signed measure for each $x \in T$ and we then have [5, p. 123] that $\lambda(x)=\lambda(x)^{+}-\lambda(x)^{-}$where $\lambda(x)^{+}, \lambda(x)^{-} \in M(S)^{+}$and $|\lambda(x)|=\lambda(x)^{+}+\lambda(x)^{-}$ for all $x \in T$. We show that $\lambda^{+}, \lambda^{-}$satisfy condition (1).

Let $f \in C_{c}(S)^{+}$and set $\mu(x, E)=\int_{E} f(y) \lambda(x, d y)$. Then for each $x, \mu(x) \in M(S)$ and for

$$
g \in C_{c}(S), \mu(g)=\int_{S} g(y) f(y) \lambda(x, d y)=\lambda(g f) .
$$

Hence $\mu(g)$ is continuous for each $g \in C_{c}(S)$ and therefore from what we have just shown $|\mu|(\cdot, S)$ is lower-semicontinuous since $S$ is open. But $|\mu|(x, S)=\int_{S} f(y)|\lambda|(x, d y)$ and therefore $|\lambda|(f)$ is lower semicontinuous for each $f \in C_{c}(S)^{+}$. Since $|\lambda|(x)=\lambda^{+}(x)+\lambda^{-}(x)$ and $\lambda(x)=$ $\lambda^{+}(x)-\lambda^{-}(x)$ it now follows that for $f \in C_{c}(S)^{+}, \lambda^{+}(f)$ and $\lambda^{-}(f)$ are lower semi-continuous. But then it follows from (1) that $\lambda^{+}(\cdot, E), \lambda^{-}(\cdot, E)$ and hence $\lambda(\cdot, E)$ are measurable for each Borel set $E$.

Condition (3) easily follows for we can approximate $\lambda(f)$ uniformly by means of measurable functions of the form $\sum_{i=1}^{n} a_{i} \lambda\left(\cdot, E_{i}\right)$.

REMARK 1. T need not be Hausdorff or locally compact in Theorem 1.

Proof of Theorem 2. It is well known that $\mu(E)=\int_{T} \lambda(x, E) \nu(d x)$ defines a measure on $S$ such that $\int_{S} f d \mu=\int_{T} \lambda(f) d \nu$ for $f \in B(S)$. Hence we will only show that $\mu$ is regular.

We can assume that $\nu$ is real and $\|\boldsymbol{\nu}\|=1$. Further we can suppose that $\lambda(x) \in M(S)^{+}$for each $x \in T$. For we can first assume that $\lambda(x)$ is a real signed measure, and writing $\lambda(x)=\lambda(x)^{+}-\lambda(x)^{-}$, the proof of Theorem 1 shows that for $f \in C_{c}(S)^{+}, \lambda^{+}(f)$ and $\lambda^{-}(f)$ are lower semi-continuous. Hence we have the condition (1) of Theorem 1 and additionally, $\|\lambda\|=\sup \{\|\lambda(x)\|: x \in S\}<\infty$. 
LEMma 1. Let $U$ be an open set in $S, \chi$ its characteristic function. Let $X=\left\{f \in C_{c}(S): 0 \leqq f \leqq \chi\right\}, Y=\left\{g \in C_{c}(T): 0 \leqq g \leqq \lambda(\cdot, U)\right\}$. Then

$$
\sup \left\{\int_{T} g d \nu: g \in Y\right\} \leqq \sup \left\{\int_{T} \lambda(f) d \nu: f \in X\right\}
$$

Proof. Let $g \in Y, \varepsilon>0$ and let $g$ vanish outside the compact set $K$ and fix $x \in K$.

Since $g \in Y$ then $g(x)-\varepsilon / 2<\lambda(x, U)$ and hence there is a function $f \in X$ such that $g(x)-\varepsilon / 2<\lambda(f)(x)$. Since $\lambda(f)$ is lower semicontinuous there is a neighborhood $V$ of $x$ such that for $t \in V$ one has $g(x)-\varepsilon / 2<\lambda(f)(t)$. But also there is a neighborhood $V^{\prime}$ of $x$ such that if $t \in V^{\prime}$ then $g(t)-\varepsilon<g(x)-\varepsilon / 2$. Hence there is a neighborhood $W$ of $x$ such that for $t \in W, g(t)-\varepsilon<\lambda(f)(t)$. We extract a finite cover of sets $W$ of $K$ with associated functions $f \in X$. If we let $h$ be the pointwise maximum of the corresponding functions $f$ then $h \in X$ and for $t \in K$ we have

$$
g(t)-\varepsilon<\lambda(h)(t) .
$$

Hence $\int_{T} g d \nu-\varepsilon<\int_{T} \lambda(h) d \nu$ and the proof is complete.

LEMmA 2. $\int_{T} \lambda(x, U) \nu(d x) \leqq \sup \left\{\int_{T} g d \nu: g \in Y\right\}$.

Proof. Let $\varepsilon>0$ and $n$ be an integer such that $n \varepsilon>\|\lambda\| \geqq$ $(n-1) \varepsilon$. Then set

$$
E_{k}=\{x \in T: k \varepsilon<\lambda(x, U) \leqq(k+1) \varepsilon\} \quad \text { for } k=0,1, \cdots, n-1 .
$$

Then $\left\{E_{k}\right\}$ is a partition of $T$ by Borel sets and

$$
0 \leqq \int_{T} \lambda(x, U) \nu(d x)-\sum_{k=0}^{n-1} k \varepsilon \nu\left(E_{k}\right)<\varepsilon .
$$

Let

$$
U_{k}=\{x: \lambda(x, U)>k \varepsilon\} .
$$

Then $U_{k}$ is an open set and $E_{k}=U_{k}-U_{k+1}$. Since $\nu$ is regular then for each $k$ there is a compact set $K_{k} \subset E_{k}$ such that $\nu\left(E_{k}-K_{k}\right)<\varepsilon / n^{2}$. We can then find for each $k$ an open set $V_{k}$ with compact closure contained in $U_{k}$ and containing $K_{k}$. Further there exist functions $f_{k} \in C_{c}(T)^{+}$for $k=0, \cdots, n-1$ such that $f_{k}(x)=k \varepsilon$ for $x \in K_{k}, f_{k}(x)=0$ for $x \in T-V_{k}$ and $0 \leqq f_{k}(x) \leqq k \varepsilon$ for all $x \in T$. Therefore $f_{k}(x) \leqq$ $k \varepsilon<\lambda(x, U)$ for $x \in U_{k}$ and hence $f_{k} \in Y$. We let 


$$
f(x)=\max \left\{f_{k}(x): 0 \leqq k \leqq n-1\right\} .
$$

It follows that $f \in Y$ and

$$
f(x) \leqq \sum_{k=0}^{n-1} k \varepsilon \chi_{k}(x),
$$

where $\chi_{k}$ denotes the characteristic function of the set $E_{k}$. We then have

$$
\begin{aligned}
0 & \leqq \int_{T} \sum_{0}^{n-1} k \varepsilon \chi_{k} d \nu-\int_{T} f d \nu \\
& \leqq \sum_{0}^{n-1} \int_{E_{k}}\left(k \varepsilon-f_{k}\right) d \nu \\
& =\sum_{0}^{n-1} \int_{E_{k}-\boldsymbol{K}_{k}}\left(k \varepsilon-f_{k}\right) d \nu \\
& \leqq \sum_{0}^{n-1} \int_{E_{k}-\boldsymbol{K}_{k}} k \varepsilon d \nu \\
& \leqq \sum_{0}^{n-1} k \varepsilon^{2} / n^{2} \leqq \varepsilon^{2}
\end{aligned}
$$

But

$$
\int_{T} \sum_{0}^{n-1} k \varepsilon \chi_{k} d \nu=\sum_{0}^{n-1} k \varepsilon \nu\left(E_{k}\right)
$$

and applying (1) we have

$$
0 \leqq \int_{T} \lambda(x, U) \nu(d x)-\int_{T} f d \nu \leqq \varepsilon^{2}+\varepsilon
$$

completing the proof.

LEMma 3. $\mu(U)=\sup \left\{\int_{S} f d \mu: f \in X\right\}$ and $\mu$ is regular.

Proof. Combining Lemma 1 and Lemma 2 we have

$$
\mu(U) \leqq \sup \left\{\int_{T} \lambda(f) d \nu: f \in X\right\}
$$

$\operatorname{But} \int_{S} f d \mu=\int_{T} \lambda(f) d \nu$ and therefore

$$
\mu(U) \leqq \sup \left\{\int_{s} f d \mu ; f \in X\right\} \leqq \mu(U)
$$

Now the mapping $f \rightarrow \int_{S} f d \mu$ defines a bounded linear form on the space $C_{0}(S)$ and hence there is a measure $\omega \in M(S)^{+}$such that $\int_{S} f d \mu=\int_{S} f d \omega$ for all $f \in C_{0}(S)$ and since $\omega$ is regular 


$$
\omega(U)=\sup \left\{\int_{s} f d \omega: f \in X\right\}=\mu(U) .
$$

This means the collection $\Sigma$ of all Borel sets $E$ for which $\omega(E)=\mu(E)$ contains all open sets and it follows from [6, p. 2] as in the proof of (1) Theorem 1 that $\Sigma$ is the class of all Borel sets. Hence $\mu$ is the regular measure $\omega$. It is easily seen that $|u|(E) \leqq \int_{T}|\lambda|(x, E)|\nu|(d x)$ and the proof is complete.

Proof of Theorem 3. From [1], [4] and the Riesz Representation Theorem, $X^{*}=M(S)$ and $Y^{*} \supset M(T)$. From [9, pp. 38-39]

$$
A^{*}(M(T)) \supset M(S)
$$

and the formula $\lambda(x)=A^{*} \stackrel{\circ}{x}$, where $\stackrel{\circ}{x}(E)=1$ if $x \in E$, 0 if $x \notin E$, defines a map $\lambda: T \rightarrow M(S)$ satisfying (3) of Theorem 1 since $\|\lambda\|=$ $\sup \left\{\|A f\|:\|f\| \leqq 1, f \in C_{0}(S)\right\}<\infty$ because the norm, $\beta$ and $\beta^{\prime}$ bounded sets are the same (see [1] and [4]) and from [9, p. 45] $A$ takes bounded sets into bounded sets. Furthermore $A f=\lambda(f)$ for $f \in X$ and if $\nu(E)=\int_{T} \lambda(x, E) \mu(d x)$ then

$$
\int_{S} f d \nu=\int_{T} \lambda(f) d \mu=\int_{T} A f d \mu=\int_{S} f d\left(A^{*} \mu\right)
$$

for all $f \in X$ and consequently $A^{*} \mu=\nu$ since $\nu$ is regular. Finally if $A^{* *}$ is the adjoint of $A^{*}$ restricted to $M(T)$ then for $\mu \in M(T)$ and

$$
f \in B(S)\left[A^{* *} f\right](\mu)=f\left(A^{*} \mu\right)=\int_{S} f d\left(A^{*} \mu\right)=\int_{T} \lambda(f) d \mu=[\lambda(f)](\mu)
$$

since $\lambda(f) \in B(T)$. This holds for all $u \in M(T)$ and consequently $A^{* *} f=\lambda(f)$. Hence $A^{* *}(B(S)) \subset B(T)$ and $\left\|A^{* *}\right\|=\|\lambda\|$.

REMARK 2. If for each $t \in[0, \infty], T(t)$ is a continuous operator from $X$ to $X$ and $T(t+u)=T(t) T(u)$ then $T(t+u)^{* *}=T(t)^{* *} T(u)^{* *}$. If we then write $[T(t) f](x)=\int_{S} f(y) \lambda_{t}(x, d y)$, then by the above theorem $\lambda_{t}(f)=T(t)^{* *} f$ for $f \in B(S)$. If $\chi$ is the characteristic function of the Borel set $E$ we have

$$
\lambda_{t+u}(\chi)=\lambda_{t}\left(\lambda_{u}(\chi)\right)
$$

or the Chapmann-Kolmogorov equation

$$
\lambda_{t+u}(x, E)=\int_{S} \lambda_{u}(y, E) \lambda_{t}(x, d y) .
$$

Consequently a transition function $\lambda_{t}(x, \cdot)$ can be obtained for a semi- 
group of $\beta$ or $\beta^{\prime}$ continuous operators on the space $C(S)$ when $S$ is locally compact.

REMARK 3. One can obtain a kernel $\lambda$ satisfying (1) under the weaker condition that $A$ have range $B(T)$ and domain $C_{0}(S)$. For the set of linear mappings $f \rightarrow \lambda(f)(x)$ for $x \in T$ is pointwise bounded and hence uniformly bounded since $C_{0}(S)$ is a Banach space.

Proof of Theorem 4. For each compact set $K \subset S$ there is a function $\varphi_{K} \in C_{c}(S)$ such that $\varphi_{K} \equiv 1$ on $K$. If $f \in C(S)$ then the net $\left\{\varphi_{K} f\right\} \subset C_{c}(S)$ converges $\beta^{\prime}$ to $f$ since it is uniformly bounded and $\beta$ convergent to $f$. Consequently $C_{c}(S)$ is $\beta^{\prime}$ dense in $C(S)$. If $x \in T$ and $U$ is a neighborhood of $x$ with compact closure then $\left\{\lambda\left(x_{\alpha}\right): x_{\alpha} \in U\right\}$ is a $\beta^{\prime}$-equicontinuous set of linear functionals on $C(S)$ for any net $\left\{x_{\alpha}\right\} \subset U$ converging to $x$. By hypothesis $\lambda\left(x_{\alpha}\right) \rightarrow \lambda(x)$ on $C_{c}(S)$. Since $C_{c}(S)$ is $\beta^{\prime}$ dense and $\left\{\lambda\left(x_{\alpha}\right)\right\}$ is $\beta^{\prime}$-equicontinuous, $\lambda\left(x_{\alpha}\right) \rightarrow \lambda(x)$ on $C(S)$. Hence $\lambda(f)$ is continuous at $x$ for all $f \in C(S)$.

Conversely if $\lambda(f) \in C(T)$ for $f \in C(S)$ then for any compact set $K \subset T\{\lambda(x): x \in K\}$ is weak-* compact as as ubset of the dual of $C(S)_{\beta}$ and, as Conway [3] has shown, must be $\beta$-equicontinuous.

Proof of Theorem 5. Suppose that $A$ is continuous from $C(S)_{\beta}$, to $C(T)_{\beta \prime}$ or $C(T)_{\beta}$. Then $\|\lambda\|<\infty$ by Theorem 3 and if $K$ is a compact set in $T$ and $V$ is the $\beta$ neighborhood of 0 defined by some function $\varphi \in C_{0}(T)$ identically 1 on $K$ there is a $\beta^{\prime}$ neighborhood of $0, U$, such that $A(U) \subset V$. That is, $|\lambda(f)(x)| \leqq 1$ for all $f \in U$ and $x \in K$. Consequently $\lambda$ satisfies condition $E^{\prime}$.

Conversely, let us show $A$ is continuous from $C(S)_{\beta}$, into $C(T)_{\beta}$, Let $V$ be a $\beta^{\prime}$ neighborhood of 0 in $C(T)$ and $r>0$. We show there is a $\beta$ neighborhood $U$ of 0 in $C(S)$ such that $A^{-1}(V) \supset B_{r} \cap U$ thus showing that $A^{-1}(V)$ is a $\beta^{\prime}$ neighborhood.

Let $p=r\|\lambda\|$. There is a $\phi \in C_{0}(T)$ such that

$$
V \supset B_{p} \cap\left\{g: P_{\phi}(g) \leqq 1\right\} \text { and } \phi \geqq 0 \text {. }
$$

Let $K=\{t:|\phi(t)| \geqq 1 /(p+1)\}$. Since $\lambda$ satisfies condition $E^{\prime}$ there is a $\beta^{\prime}$ neighborhood $U_{0}$ in $C(S)$ such that $|\lambda(f)(x)| \leqq 1$ for all $f \in U_{0}$ and $x \in K$. Let $W=\{f \in C(S):\|\phi\| f \in U\}$. Then $A^{-1}(V) \supset B_{r} \cap W$ for if $f \in B_{r} \cap W$ then $A f \in B_{p}$ and $|\phi(x)[A f](x)|<p /(p+1)$ for $x \notin K$ while for $x \in K,|\phi(x)[A f](x)| \leqq\|\phi\||[A f](x)| \leqq 1$ since $\|\phi\| f \in U_{0}$. Hence

$$
A^{-1}(V) \supset A^{-1}\left(B_{p}\right) \cap A^{-1}\left\{g: P_{\phi}(g) \leqq 1\right\} \supset B_{r} \cap\left(B_{r} \cap W\right)=B_{r} \cap W .
$$

We then choose a $\beta$ neighborhood $U$ such that $W \supset B_{r} \cap U$ completing the proof. 
REMARK 4. If $A$ is continuous from $C(S)_{\beta}$ into $C(T)_{\beta}$, it follows that $\lambda$ satisfies $E$.

The proof of Corollary 1 is almost immediate. As a consequence of Theorem 3 and Theorem 5 continuity from $C(S)_{\theta}$, to $C(T)_{\beta}$, is equivalent to condition $E^{\prime}$. If $A^{\prime}$ is an extension of $A$ to $C(S)$ into $C(T)$ given by a kernel $\mu$ then $\mu=\lambda$ on $C_{0}(S)$ and consequently $\mu=\lambda$ on $C(S)$ and $A=A^{\prime}$. Since by Theorem 3 any $\beta$ or $\beta^{\prime}$ continuous extension is given by a kernel this shows that $A^{* *}$ is unique.

It should be noted that if $S$ is paracompact and $A$ is any operator on $C(S)$ into $C(T)$ given by a bounded kernel $\lambda$ then by Theorems 4 and $5, A$ is continuous from $C(S)_{\beta}$ to $C(T)_{\beta^{\prime}}$.

We conclude with a brief remark on operators from $M(T)$ into $M(S)$. Suppose $B$ is such a linear operator and $B^{*}$ its adjoint on $B(S)$. Define $\lambda: T \rightarrow M(S)$ by $\lambda(x)=B \stackrel{\circ}{x}$ where $\stackrel{\circ}{x}$ is the measure defined in the proof of Theorem 3 . If $B$ is bounded and $B^{*}\left(C_{c}(S)\right) \subset C(T)$ then $B^{*}(B(S)) \subset B(T)$ by Theorem 1 . By Theorem 2,

$$
(B \mu)(E)=\int_{T}(B \stackrel{\circ}{x})(E) \mu(d x) .
$$

If $\lambda$ satisfies condition $E^{\prime}$ then by Theorem $5 B$ is the adjoint of the continuous operator $B^{*}$ from $C(S)_{\beta}$, to $C(T)_{\beta}$. Thus $B$ is completely determined by its action on the point measures $\{\dot{x}: x \in T\}$.

REMARK 5 (added January 13, 1967). One can amplify Remark 4 by observing that if, moreover, $\lambda$ satisfies $E$ then Theorem 5 remains true with $\beta^{\prime}$ replaced by $\beta$. For then $A$ is continuous from $C(S)_{\beta}$, to $C(T)_{\beta}$ and using condition $E$, [3], part (2) of Theorem 3 and [9, p. $39]$ it follows that $A^{*}$ takes $\beta$-equicontinuous sets of $M(T)$ into $\beta$ equicontinuous sets of $M(S)$ making $A$ continuous on $C(S)_{\beta}$ into $C(T)_{\beta}$.

REMARK 6. It has recently come to the author's attention that a version of Theorem 2 can be found on page 176 of the recent book by P. A. Meyer, Probability and Potentials, Blaisdell, Waltham, Massachusetts, 1966, under the conditions that $S$ be $\sigma$-compact, $\lambda: S \rightarrow M(S)^{+}, \lambda(f)$ be continuous for all $f \varepsilon C_{c}(S)^{+}$and that $\nu$ have compact support.

\section{REFERENCES}

1. R. C. Buck, Bounded continuous functions on a locally compact space, Michigan Math. J. 5 (1958), 95-104.

2. H. S. Collins and J. R. Dorroh, Remarks on certain function spaces (to appear in Math. Ann.)

3. J. B. Conway, The strict topology and compactness in the space of measures, Bull. Amer. Math. Soc. 72 (1966), 75-78. 
4. J. R. Dorroh, Semi-groups of maps in a locally compact space (to appear in Canad. J. Math.)

5. N. Dunford and J. T. Schwartz, Linear Operators, Interscience, New York, 1958.

6. E. Dynkin, Die Grundlagen der Theorie der Markoffschen Prozesse, Springer, Berlin, 1961.

7. S. R. Foguel, Existence of invariant measures for Markov Processes II, Proc. Amer. Math. Soc. 17 (1966), 387-89.

8. J. L. Kelley, General Topology, D. Van Nostrand, New York, 1955.

9. A. P. and W. J. Robertson, Topological Vector Spaces, Cambridge Tracts in Math. and Math. Physics, No. 53, Cambridge University Press, London, 1964.

Received August 8, 1966. This material is a result of the author's dissertation research under the direction of Dr. J. R. Dorroh to whom the author is especially grateful.

LOUISIANA STATE UNIVERSity

BATON ROUge, LOUISIANA 


\section{PACIFIC JOURNAL OF MATHEMATICS}

\section{EDITORS}

\section{H. ROYDEN}

Stanford University

Stanford, California

J. P. JANS

University of Washington

Seattle, Washington 98105

\section{J. DUGUNDJI}

Department of Mathematics

Rice University

Houston, Texas 77001

RICHARD ARENS

University of California

Los Angeles, California 90024

ASSOCIATE EDITORS

E. F. BECKENBACH

B. H. NeumanN

F. WoLF

K. YosidA

\section{SUPPORTING INSTITUTIONS}

\author{
UNIVERSITY OF BRITISH COLUMBIA \\ CALIFORNIA INSTITUTE OF TECHNOLOGY \\ UNIVERSITY OF CALIFORNIA \\ MONTANA STATE UNIVERSITY \\ UNIVERSITY OF NEVADA \\ NEW MEXICO STATE UNIVERSITY \\ OREGON STATE UNIVERSITY \\ UNIVERSITY OF OREGON \\ OSAKA UNIVERSITY \\ UNIVERSITY OF SOUTHERN CALIFORNIA
}

\author{
STANFORD UNIVERSITY \\ UNIVERSITY OF TOKYO \\ UNIVERSITY OF UTAH \\ WASHINGTON STATE UNIVERSITY \\ UNIVERSITY OF WASHINGTON \\ AMERICAN MATHEMATICAL SOCIETY \\ CHEVRON RESEARCH CORPORATION \\ TRW SYSTEMS \\ NAVAL ORDNANCE TEST STATION
}




\section{Pacific Journal of Mathematics}

\section{Vol. 23, No. 1 \\ March, 1967}

M. J. C. Baker, A spherical Helly-type theorem ................... 1

Robert Morgan Brooks, On locally m-convex*-algebras.............. 5

Lindsay Nathan Childs and Frank Rimi DeMeyer, On automorphisms of separable algebras ...................................

Charles L. Fefferman, A Radon-Nikodym theorem for finitely additive set

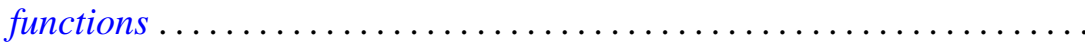

Magnus Giertz, On generalized elements with respect to linear

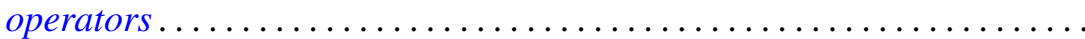

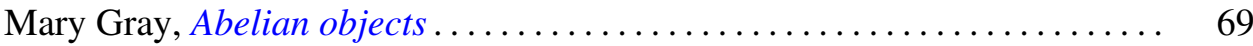

Mary Gray, Radical subcategories.............................. 79

John A. Hildebrant, On uniquely divisible semigroups on the two-cell . . . . . 91

Barry E. Johnson, AW*-algebras are $\mathrm{QW}^{*}$-algebras ............... 97

Carl W. Kohls, Decomposition spectra of rings of continuous functions . . . . 101

Calvin T. Long, Addition theorems for sets of integers .............. 107

Ralph David McWilliams, On $w^{*}$-sequential convergence and quasi-reflexivity ................................... 113

Alfred Richard Mitchell and Roger W. Mitchell, Disjoint basic

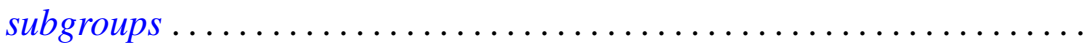

John Emanuel de Pillis, Linear transformations which preserve hermitian and positive semidefinite operators .

Qazi Ibadur Rahman and Q. G. Mohammad, Remarks on Schwarz's lemma

Neal Jules Rothman, An $L^{1}$ algebra for certain locally compact topological semigroups ...

F. Dennis Sentilles, Kernel representations of operators and their adjoints ...

D. R. Smart, Fixed points in a class of sets

K. Srinivasacharyulu, Topology of some Kähler manifolds

Francis C.Y. Tang, On uniqueness of generalized direct decompositions .

171 Albert Chapman Vosburg, On the relationship between Hausdorff dimension and metric dimension . 